\title{
Bem-estar psicológico: definição, avaliação e principais correlatos ${ }^{1}$
}

\author{
Psychological well-being: definition, assessment \\ and principal correlates
}

\author{
Wagner de Lara MACHADO² \\ Denise Ruschel BANDEIRA²
}

\begin{abstract}
Resumo
O bem-estar psicológico é um construto baseado na teoria psicológica a respeito do funcionamento positivo ou ótimo. Os pontos de convergência entre definições provenientes de teorias do desenvolvimento humano, psicologia humanista-existencial e saúde mental constituem suas dimensões: autoaceitação, relações positivas com outros, autonomia, domínio sobre o ambiente, propósito na vida e crescimento pessoal. O objetivo deste artigo é apresentar o conceito de bem-estar psicológico a partir de uma revisão seletiva da literatura. São examinados as origens e o desenvolvimento do conceito, bem como as abordagens na sua avaliação. Correlatos psicossociais, sociodemográficos e neurobiológicos do bem-estar psicológico são brevemente descritos e discutidos. O bem-estar psicológico está associado a processos positivos relacionados à saúde. Aponta-se a necessidade de estudos sobre o construto no Brasil.
\end{abstract}

Unitermos: Bem-estar psicológico. Bem-estar subjetivo. Saúde mental.

\begin{abstract}
Psychological well-being is a construct based on psychological theory about positive or optimal functioning. The convergence points between definitions from theories of human development, humanistic-existential psychology and mental health are its dimensions: self-acceptance, positive relations with others, autonomy, environmental mastery, purpose in life and personal growth. The aim of this paper is to present the concept of psychological well-being from a selective literature review. The origins and development of the concept and approaches to their assessment are examined. Psychosocial, sociodemographic and neurobiological correlates of psychological well-being are briefly described and discussed. psychological well-being is associated with positive processes related to health. It indicates the need for studies about the construct in Brazil.
\end{abstract}

Uniterms: Psychological well-being. Subjective well-being. Mental health.

O Bem-Estar Psicológico (BEP) é um construto baseado na teoria psicológica, agregando conhecimentos de áreas como psicologia do desenvolvimento humano, psicologia humanista-existencial e saúde mental, a respeito do funcionamento psicológico positivo ou ótimo (Ryff, 1989). Os pontos de convergência

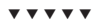

1 Artigo elaborado a partir da dissertação de W.L. MACHADO, intitulada"Escala de bem-estar psicológico: adaptação para o português brasileiro e evidências de validade". Universidade Federal do Rio Grande do Sul, 2010.

2 Universidade Federal do Rio Grande do Sul, Instituto de Psicologia, Programa de Pós-Graduação em Psicologia. R. Ramiro Barcelos, 2600, Santana, 90035003, Porto Alegre, RS. Correspondência para/Correspondence to: W.L. MACHADO. E-mail: <wag_psico@yahoo.com.br>. 
entre essas formulações teóricas constituem as dimensões do BEP: autoaceitação, relações positivas com outros, autonomia, domínio sobre o ambiente, propósito na vida e crescimento pessoal (Ryff,1989; Ryff \& Keyes, 1995; Ryff \& Singer, 2008). Diversos estudos têm demonstrado sua convergência em relação a indicadores de bem-estar, qualidade de vida, marcadores biológicos de saúde, processos desenvolvimentais adaptativos e construtos que refletem dimensões positivas da saúde mental (Ryff \& Singer, 2008).

O objetivo do presente artigo é apresentar o conceito de BEP, examinando suas origens, desenvolvimento e abordagens de avaliação. Algumas considerações teóricas a respeito do BEP e indicadores prévios de bem-estar também serão expostos. Achados em relação ao BEP e correlatos psicossociais, sociodemográficos e neurobiológicos serão brevemente descritos e discutidos, a partir de uma revisão seletiva dessas investigações.

\section{Origens do estudo do bem-estar no âmbito psicológico}

A questão sobre o que constitui o bem-viver tem sido um tema central na civilização ocidental desde os antigos filósofos gregos. Contudo, a inclusão do estudo do bem-estar no âmbito psicológico no domínio da investigação científica deu-se apenas na década de 1960, impulsionada por grandes transformações sociais (por exemplo o fim da Segunda Guerra Mundial) e pela necessidade de desenvolver indicadores sociais de qualidade de vida (Diener, 1984; Galinha \& Ribeiro, 2005; Keyes, 2006; Ryff, 1989). Durante esse período, psicólogos sociais e do comportamento perceberam que, até então, tinhamse explorado a fundo questões sobre as doenças mentais e o sofrimento humano, porém sabia-se muito pouco sobre aspectos como a saúde mental e a felicidade (Diener, 1984; Ryff, 1989). Diversos termos eram empregados nessas pesquisas, tais como: felicidade, satisfação, estado de espírito, moral, afeto positivo, avaliação subjetiva da qualidade de vida, entre outros (Diener, 1984).

O trabalho de Diener (1984) é um marco na tentativa de sistematização dos estudos na área, cunhando o termo Bem-Estar Subjetivo (BES) para representá-la. $O$ BES é definido como um conjunto de fenômenos que incluem respostas emocionais, domínios de satisfação e julgamentos globais de satisfação de vida. Existem, entretanto, três componentes básicos do BES: satisfação de vida, altos níveis de afeto positivo e baixos níveis de afeto negativo (Bradburn, 1969; Diener, 1984; Diener, Suh \& Oishi, 1997; Diener, Suh, Lucas \& Smith, 1999).

Décadas de pesquisas sobre o BES e seus componentes acumularam importantes achados para o estudo do bem-estar. Por exemplo, descobriu-se que os afetos positivos e negativos formam dimensões independentes (Bradburn, 1969), e a satisfação de vida tende a ser o componente mais estável ao longo do tempo (Diener et al., 1999). Sobre a mensuração do BES, a frequência (e não a intensidade) dos níveis de afeto constitui seu melhor indicador (Diener, 1984; Diener et al., 1999), e escalas de múltiplos itens são mais fidedignas do que aquelas de item único (Diener et al., 1999; Larsen, Diener \& Emmons, 1985). Ainda, os traços de personalidade extroversão e neuroticismo aparecem como grandes preditores do BES (Costa \& McCrae, 1980).

Em resumo, a pesquisa na área avançou consideravelmente em termos da mensuração do BES e dos fatores que influenciam em sua variabilidade. Contudo, a tarefa essencial de definir a estrutura básica do bem-estar no âmbito psicológico foi negligenciada nesses estudos (Ryff, 1989; Ryff \& Keyes, 1995).

\section{O bem-estar psicológico}

O modelo do BEP surgiu no final da década de 1980, com o trabalho de Ryff (1989) na tentativa de definir a estrutura básica do bem-estar no âmbito psicológico. A autora critica a falta de uma consistente base teórica que justifique a escolha dos indicadores do BES. Estudos prévios que utilizaram medidas de afetos positivos e negativos e satisfação de vida tinham como propósito investigar a influência de mudanças sociais (Bradburn, 1969) e do envelhecimento bem-sucedido (Neugarten, Havighurst \& Tobin, 1961) no desenvolvimento, e não a essência do bem-estar psicológico. Além disso, a interpretação da felicidade como sendo a principal motivação da existência humana incide sobre a interpretação equivocada da obra de filósofos clássicos, como os escritos de Aristóteles (Ryff, 1989; Ryff \& Keyes, 1995).

Aristóteles em sua doutrina ética propõe que o bem-viver resulta da eudaimonia, isto é, provém da ação em direção ao desenvolvimento dos potenciais únicos 
de cada pessoa (Ryff, 1989; Waterman, 1993). Consequentemente, a eudaimonia está relacionada às experiências de desenvolvimento pessoal, autorrealização e sentido de vida (Ryan \& Deci, 2001; Ryff \& Singer, 2008; Waterman, 1993). Formulações sobre a essência do bem-estar em termos de experiências de prazer, contentamento, satisfação e felicidade (isto é, BES) se assemelham à concepção hedônica (por exemplo: Aristipo de Cirene) do bem-viver, em que este provém da satisfação dos desejos, da busca pelo prazer e da evitação da dor (Ryan \& Deci, 2001; Waterman, 1993). A expressão grega eudaimonia fora equivocadamente traduzida por felicidade, desconsiderando diferenças fundamentais entre hedonismo e eudaimonismo, e suas implicações teóricas no estudo do bem-estar (Ryan \& Deci, 2001; Ryff, 1989; Waterman, 1993).

Em sua busca ao retorno à concepção do bem-estar por meio da eudaimonia, Ryff (1989) realizou uma extensa revisão da literatura a respeito do funcionamento ótimo ou positivo no âmbito psicológico. Os pontos de convergência entre definições de teorias humanista-existenciais, do desenvolvimento humano e da saúde mental, compuseram as dimensões do construto. A Tabela 1 apresenta os autores, os conceitos-chave e as seis dimensões baseadas na teoria psicológica do BEP.

Assim, as características que constituem a essência do BEP incluem: possuir uma atitude positiva em relação a si mesmo e aceitar múltiplos aspectos de sua personalidade (autoaceitação); possuir relacionamentos acolhedores, seguros, íntimos e satisfatórios com outras pessoas (relações positivas com outros); ser autodeterminado, independente, avaliar experiências pessoais segundo critérios próprios (autonomia); ter competência em manejar o ambiente para satisfazer necessidades e valores pessoais (domínio sobre o ambiente); ter senso de direção, propósito e objetivos na vida (propósito na vida); perceber um contínuo desenvolvimento pessoal e estar aberto a novas experiências (crescimento pessoal) (Ryff,1989; Ryff \& Keyes, 1995; Ryff \& Singer, 2008).

\section{A avaliação do bem-estar psicológico}

A partir da definição do construto, Ryff (1989) iniciou o desenvolvimento de escalas psicométricas destinadas à avaliação das seis dimensões do BEP. Cada dimensão foi operacionalizada em termos de escores positivos e negativos, e três juízes previamente treinados formularam 80 itens para cada dimensão. Esses itens deveriam ser escritos na forma de autodescrições que refletissem as definições teóricas subjacentes ao construto e aplicáveis a adultos de ambos os sexos e de qualquer faixa etária. Análises preliminares permitiram descartar itens que poderiam apresentar baixo desempenho psicométrico, ocasionando a retenção de 32 itens por dimensão.

Estes itens foram aplicados a uma amostra de 312 adultos jovens, de meia idade e idosos. A partir da matriz de correlações entre os itens, foram descartados aqueles que apresentassem baixa correlação com sua dimensão original, e que eventualmente se correlacionassem com outras dimensões. Ao final, foram mantidos 20 itens por dimensão (10 positivos e 10 negativos). As escalas demonstraram um bom desempenho psicométrico, apresentando medidas de fidedignidade Alpha de Cronbach entre 0,86 e 0,93. Análises fatoriais de se-

Tabela 1. As bases teóricas e dimensões do bem-estar psicológico.

\begin{tabular}{lll}
\hline Autores & Conceitos-chave & Dimensões do Bem-estar Psicológico \\
\hline Allport & Maturidade & Autoaceitação \\
Neugarten & Mudanças na personalidade no ciclo vital & Propósito na vida \\
Buhler & Tendências básicas da vida & Domínio sobre o ambiente \\
Erikson & Estágios psicossociais & Relações positivas com outros \\
Frankl & Sentido na vida & Crescimento pessoal \\
Jahoda & Saúde mental & Autonomia \\
Jung & Individuação & \\
Maslow & Autoatualização & \\
Rogers & Pessoa em funcionamento pleno & \\
\hline
\end{tabular}


gunda ordem permitiram constatar que as dimensões crescimento pessoal, relações positivas com outros, propósito na vida e autonomia nitidamente se diferenciam de indicadores prévios do bem-estar.

Posteriormente, versões alternativas e mais breves da Escala de Bem-Estar Psicológico (EBEP) foram desenvolvidas. Uma versão de 84 itens (14 por dimensão, Ryff \& Essex, 1992) é a mais utilizada em estudos do grupo de Ryff. Outra versão, de 18 itens (três por dimensão, Ryff \& Keyes, 1995), foi construída para ser utilizada em grandes levantamentos, por questão de brevidade e economia. Por fim, uma versão de 54 itens (nove por dimensão) vem sendo utilizada no Wisconsin Longitudinal Study pela equipe de Ryff (van Dierendonk, 2005). Todas as versões da EBEP têm demonstrado bons parâmetros psicométricos e correlações elevadas com a escala original de Ryff (1989). Essas escalas foram adaptadas e/ou traduzidas em diversos países, incluindo Itália (Ruini, Ottolini, Rafanelli, Ryff \& Fava, 2003), Grécia (Vleioras \& Bosma, 2005), Austrália (Burns \& Machin, 2009), China (Cheng \& Chan, 2005), Canadá (Clarke, Marshall, Ryff \& Wheaton, 2001), Alemanha (Fleeson \& Baltes, 1996), Coreia do Sul (Jin \& Moon, 2006; Keyes \& Ryff, 2003), Portugal (Novo, 2003), Suécia (Lindfors \& Lundberg, 2002), e Espanha (van Dierendonck, Díaz, Rodríguez-Carvajal, Blanco \&Moreno-Jiménez, 2007).

Diversos estudos examinaram a estrutura fatorial do modelo multidimensional do BEP, utilizando técnicas de análise fatorial confirmatória. Alguns desses estudos corroboram o modelo teórico de seis dimensões (Cheng \& Chan, 2005; Clarke et al., 2001; van Dierendonck et al., 2007), enquanto outros indicam melhor ajuste quando adicionado um fator de segunda ordem, composto pelas seis dimensões do construto (Lindfors, Berntsson \& Lundberg, 2006; van Dierendonck, 2005; Ryff \& Keyes, 1995).

Apenas o estudo realizado por Kafka e Kozma (2002) não corroborou o modelo de seis dimensões. Contudo, esse estudo apresenta duas limitações metodológicas. A primeira diz respeito ao número pequeno de participantes ( $n=277$ ) em relação ao número de indicadores (itens) de BEP (120). O outro ponto assenta sobre o método de análise fatorial empregado nesse estudo. Os autores realizaram uma análise de componentes principais com rotação do tipo Varimax, a qual obriga que os fatores extraídos se mantenham ortogonais. Com isso, Kafka e Kozma (2002) desconsideraram evidências de estudos anteriores que sugerem a correlação entre os fatores, aplicando uma técnica rotacional inadequada para o construto em questão (Ryff \& Singer, 2006). Desta forma, ambos os modelos, multidimensional e hierárquico, são utilizados atualmente.

Uma abordagem menos utilizada na investigação do BEP é a metodologia qualitativa do estudo de narrativas (Bauer \& McAdams, 2004a, 2004b; Bauer, McAdams \& Sakaeda, 2005). São investigadas as dimensões do BEP em narrativas autobiográficas em relação a objetivos de vida e etapas de transição ao longo do desenvolvimento. Esses estudos demonstram que a investigação sobre o BEP não necessita exclusivamente ser empreendida por meio de escalas psicométricas, enfatizando a utilidade de metodologias qualitativas nesse contexto.

\section{Correlatos psicossociaise sociodemográficos do BEP}

Desde a publicação original de Ryff (1989), diversos estudos investigaram a relação do BEP com processos desenvolvimentais adaptativos e construtos que refletem dimensões positivas da saúde mental (Ryff \& Singer, 2008). O BEP tem se correlacionado positivamente com autoestima e moral (Ryff, 1989), satisfação de vida e afetos positivos (Ryff, 1989), coping (Holland \& Holahan, 2003), geratividade (An \& Cooney, 2006; Queroz \& Neri, 2005), memórias integrativas e intrínsecas (Bauer \& McAdams, 2004a; Bauer et al., 2005), generosidade (Konow \& Earley, 2008), habilidades sociais (Segrin \& Taylor, 2007), inteligência emocional (Queroz \& Neri, 2005), valores intrínsecos (Sheldon, 2005), resiliência (Ryff, Keyes, Hughes, 2003) e metas intrínsecas de crescimento (Bauer \& McAdams, 2004b).

Nesta revisão são descritos os resultados da associação entre o BEP e três correlatos que frequentemente são investigados em relação ao construto, nomeadamente a idade, o nível educacional e a personalidade. A idade é uma variável geralmente utilizada para investigar as mudanças nos níveis do BEP em função de diferentes etapas do ciclo vital, enquanto o nível educacional proporciona comparar os níveis de BEP em razão do status socioeconômico (Ryff, 1989; Ryff \& Keyes, 1995; Ryff \& Singer, 2008). A escolha da variável 
personalidade é evidente, devido a sua contribuição significativa na explicação da variabilidade de medidas de bem-estar e saúde mental em geral (Costa \& McCrae, 1980; Diener, 1984; Diener et al., 1999; Schmutte \& Ryff, 1997).

Ryff (1989), Ryffe Keyes (1995) e Ryffe Singer (2008) compararam os níveis nas seis dimensões do BEP em homens e mulheres pertencentes a três diferentes grupos etários, adultos jovens, adultos de meia idade e idosos. Tomadas em conjunto, essas investigações sugerem que dimensões como autonomia e domínio sobre o ambiente apresentam um significativo incremento, enquanto as dimensões crescimento pessoal e propósito na vida decrescem expressivamente em relação a todas as faixas etárias. Contudo, as dimensões autoaceitação e relações positivas com outros tendem a não variar significativamente em função da posição no ciclo vital.

A questão sobre como o nível educacional influencia os níveis de BEP vem sendo investigada por Ryff e outros pesquisadores (Marmot, Ryff, Bumpass, Shipley \& Marks, 1997; Ryff, 1989; Ryff \& Singer, 2008). Os resultados, em geral, são convergentes ao evidenciar uma forte associação positiva entre todas as dimensões do BEP e o nível educacional. Os autores concluem que a experiência de autorrealização é facilitada por melhores condições econômicas e sociais (i.e., acesso a educação, lazer, serviços de saúde etc.).

Schmutte e Ryff (1997)3 investigaram a relação entre traços de personalidade no modelo dos Cinco Grandes Fatores (McCrae \& Costa, 1989) e as dimensões do BEP em duas amostras de adultos de meia idade. A primeira foi composta por 215 participantes (114 homens), e a segunda formada por seus cônjuges ( $n=139$ ). Como esperado, conforme estudos prévios (Costa \& McCrae, 1980), todas as seis dimensões do BEP estão negativamente associadas ao traço neuroticismo (amostra 1, r=-0,20 a $r=-0,70$; amostra 2, $r=-0,47$ a $r=-0,72$ ), e positivamente associadas ao traço extroversão (amostra $1, r=0,24$ a $r=0,44$; amostra 2, $r=0,29$ a $r=0,53$ ). Em relação aos demais traços de personalidade, as dimensões do BEP demonstraram padrões variados de associação. Por exemplo, o traço abertura à experiência se correlacionou positivamente com a dimensão crescimento pessoal (amostra 1,r=0,42; amostra 2, r=0,39), enquanto o traço socialização mostrou se correlacionar com todas as dimensões do BEP (amostra 1, $r=0,28$ a $r=0,52$; amostra 2, $r=0,27$ a $r=0,41$ ), exceto autonomia, exibindo correlações de pequena magnitude (amostra $1, r=0,14$ ) ou não significativas (amostra 2, $r=0,08, p>0,05$ ). Por fim, o traço realização se correlacionou positivamente com todas as dimensões (amostra 1, $r=0,31$ a $r=0,67$; amostra 2, $r=0,38$ a $r=0,65)$. Esses padrões de associação se mantiveram mesmo quando controladas as sobreposições do conteúdo das medidas utilizadas para as variáveis abertura e crescimento pessoal, socialização e relações positivas com outros, e realização e domínio sobre o ambiente $(r=0,17$ a $r=0,57)$.

Keyes, Shmotkin e Ryff (2002) exploraram a relação entre traços de personalidade e níveis de BES e BEP em dois grupos, um formado por participantes com escores altos de BES e baixos escores de BEP ( $n=655)$, e outro composto por participantes com escores altos de BEP e baixos escores de BES ( $n=699)$. Nenhuma diferença entre os grupos foi encontrada em relação aos níveis de extroversão e socialização, $F(1,1352)=1,69$, $p>0,05$ e $F(1,1352)=0,56, p>0,05$, respectivamente. Entretanto, o grupo com escores altos de BEP e baixos escores de BES apresentou maiores níveis de neuroticismo, $F(1,1352)=12,50, p<0,001$; abertura à experiência, $F(1$, $1352)=57,35, p<0,001$; e realização, $F(1,1352)=14,97$, $p<0,001$.

Existem pelos menos três importantes conclusões derivadas das investigações a respeito dos correlatos desenvolvimentais, psicossociais e sociodemográficos do BEP. A primeira sugere que com o avançar da idade, as pessoas tendem a ser mais confiantes em suas crenças e experiências pessoais, e também manejam melhor o ambiente a fim de atender a suas necessidades. Entretanto, o mesmo processo conduz a uma minimização da busca de objetivos de vida e do investimento no desenvolvimento pessoal. A segunda conclusão é que existe uma forte conexão entre o nível de desigualdade social e o BEP, já que maiores níveis de bem-estar estão presentes em pessoas que possuem melhores oportunidades e condições socioeconômicas. Por fim, no que tange à personalidade, fica evidente

$\boldsymbol{\nabla} \mathbf{\nabla} \boldsymbol{\nabla}$

3 Todas as correlações são significativas ao menos no nível de $p=0,05$, exceto quando indicado. 
que não somente os traços neuroticismo e extroversão desempenham uma influência relevante nos níveis de BEP, mas que outros traços de personalidade também se relacionam com o construto. Isso demonstra uma maior complexidade entre a associação do BEP e traços de personalidade, comparada a outros indicadores de bem-estar.

\section{Correlatos biológicos do BEP}

De acordo com Ryff e Singer (2008), uma área em crescente desenvolvimento no estudo do BEP diz respeito a seus substratos neurobiológicos. A partir de teorias consequencialistas, é testada a hipótese de que a experiência de uma vida de desenvolvimento e crescimento contínuo constitui parte do processo que mantém as pessoas saudáveis, seja por meio de melhores hábitos ligados a saúde, ou através do funcionamento ótimo dos processos fisiológicos do organismo. Essa abordagem é vista como uma alternativa ao modelo biomédico, focado na doença e enfermidade (Ryff \&Singer, 1998).

Ryff, Singer e Love (2004), por exemplo, estudaram a relação entre o BEP e diversos biomarcadores (por exemplo neuroendócrinos, imunológicos, cardiovasculares, e qualidade do sono) em 135 mulheres com idade entre 61 e 91 anos. Os resultados apontam que três dimensões estão associadas a uma melhor regulação neuroendócrina: crescimento pessoal e propósito na vida se correlacionam com o decréscimo do nível de cortisol salivar do início ao final do dia $(r=0,21$ a $r=0,29$, $p<0,05)$, enquanto maiores níveis de noradrenalina são encontrados em sujeitos com altos escores em autonomia $(r=0,29, p<0,01)$. Em relação ao sistema imunológico, altos escores na dimensão propósito na vida estão associados a níveis menores de marcadores inflamatórios (sIL-6r presente no plasma, um receptor solúvel da interleucina-6, $r=-0,27, p<0,01$ ).

A relação entre as seis dimensões do BEP e marcadores cardiovasculares ${ }^{4}$ mostram diferentes padrões de associação. Medidas do peso dos participantes se correlacionaram negativamente com a dimensão rela- ções positivas com outros ( $r=-0,21$ a $r=-0,26$,), enquanto a razão cintura/quadril se correlacionou na mesma direção com as dimensões relações positivas com outros e propósito na vida ( $r=-0,17$ a $r=-0,32)$. Os níveis de colesterol Lipoproteína de Alta Densidade (HDL, isto é colesterol "bom") correlacionaram-se positivamente com as dimensões crescimento pessoal e propósito na vida $(r=0,17$ a $r=0,22)$, ao passo que a razão colesterol total/HDL se correlacionou na direção oposta com a dimensão crescimento pessoal $(r=-0,16)$. Por fim, as dimensões relações positivas com outros, domínio sobre o ambiente e autoaceitação se correlacionaram negativamente $(r=-0,19$ a $r=-0,21)$ com níveis de hemoglobinas glicosiladas, marcador que indica resistência à insulina e risco de diabetes.

Por fim, três dimensões mostraram-se associadas a marcadores de qualidade do sono ${ }^{4}$ : o tempo total na cama, a duração do sono e o tempo de ingresso ao sono tipo Rapid Eye Movement (REM) estão positivamente correlacionados à dimensão domínio sobre o ambiente ( $r=0,25, r=0,22$ e $r=-0,28$, respectivamente). As dimensões relações positivas com outros e propósito na vida estão negativamente correlacionadas à movimentação ao dormir ( $r=-0,35$ e $r=-0,30$, respectivamente). Já a duração do sono REM está positivamente associada às dimensões relações positivas com outros e domínio sobre o ambiente ( $r=0,32$ e $r=0,19$, respectivamente).

A relação entre o sistema imune e o BEP também foi investigada por Hayney et al. (2006), a partir da mensuração da produção induzida de citocinas, através de vacinação, em 18 participantes (9 mulheres). A concentração sobrenadante de interferona- $\boldsymbol{\gamma}(\mathrm{IFN}-\boldsymbol{\gamma})$ e interleucina-10 (IL-10) foram medidas em culturas celulares $\left(10^{5} / 100 \mu \mathrm{L}\right)$, após a estimulação de antígenos dos vírus influenza e da hepatite A, nos períodos de 14 e 28 dias. Uma medida composta do BEP (somatório dos escores das seis dimensões) mostrou correlacionar-se positivamente com a concentração de IFN- $\gamma$ antes da estimulação $(r=0,59, p=0,03)$ e aproximou-se do nível de significância após 28 dias nas amostras que receberam antígenos da hepatite $\mathrm{A}(r=0,55, p=0,07)$. Essa mesma medida mostrou correlacionar-se positivamente

592 Todas as correlações são significativas ao menos no nível de $p=0,05$. 
com a concentração de IL-10 antes da estimulação $(r=0,67, p=0,01)$, após 28 dias da estimulação com antígenos de influenza $(r=0,57, p=0,04)$ e aproximou-se do nível de significância após 28 dias da estimulação com antígenos do vírus da hepatite $\mathrm{A}(r=0,54, p=0,08)$.

Lindfors e Lundberg (2002) investigaram a associação do nível de cortisol salivar e BEP em 26 funcionários públicos suecos (14 homens) com idade entre 24 e 62 anos. A dimensão propósito na vida correlacionou-se negativamente $(r=-0,44, p<0,05)$ com a média de concentração de cortisol salivar (no período de dois dias), enquanto a dimensão autoaceitação correlacionou-se negativamente $(r=-0,46, p<0,05)$ com a concentração de cortisol salivar no período da manhã. Ainda, a dimensão domínio sobre o ambiente correlacionou-se negativamente com ambos os indicadores ( $r=-0,56, p<0,01$ e $r=-0,64, p<0,001$, respectivamente).

Urry et al. (2004) estudaram a relação entre os padrões de ativação do córtex frontal e pré-frontal e os níveis de BEP em 84 participantes (43 homens), destros, com idade entre 57 e 60 anos. Utilizando técnicas de Eletroencefalografia (EEG), eles encontraram associação entre cinco dimensões do BEP (exceto autonomia) e padrões de assimetria cerebral (isto é, maior ativação do hemisfério esquerdo em relação ao direito), com correlações variando de $r=0,22$ a $r=0,39(p<0,05$, unicaudal). Esse padrão de assimetria cerebral foi previamente associado a estilos disposicionais positivos e ao menor risco de desenvolver sintomas depressivos.

Examinando o conjunto desses achados, emergem evidências que corroboram a hipótese de que o BEP correlaciona-se com diversos marcadores neurobiológicos de saúde. Contudo, uma questão surge naturalmente: a associação entre essas variáveis é explicada pelos níveis de bem-estar propriamente, ou refletem simplesmente relações de direção oposta daquelas em relação a indicadores de doença mental? Um exemplo talvez torne mais clara essa proposição: a associação das dimensões crescimento pessoal e propósito na vida com os níveis de cortisol salivar no estudo de Ryff et al. (2004) pode dever-se à ausência de sintomas de doença mental depressão, ansiedade etc.) na amostra estudada. O tema evoca o debate, principalmente discutido por Keyes (2002, 2007), se as dimensões positivas e negativas da saúde mental formam um único continuum bipolar (isto é, a ausência de uma indica necessariamente a presença de outra) ou se constituem dimensões independentes, porém correlacionadas (isto é, compartilham certa variabilidade, porém possuem padrões singulares de associação com outras variáveis). O autor defende a última hipótese, atualmente corroborada por uma quantidade razoável de evidências empíricas (Keyes, 2007).

Ryff et al. (2006) testaram a hipótese de que dimensões positivas e negativas da saúde mental possuem diferentes correlatos biológicos, em uma amostra de 135 mulheres, com idade entre 61 e 91 anos $(M=74$, $\mathrm{DP}=7,08)$. As medidas da dimensão positiva incluíram a EBEP e duas escalas de afetos positivos, enquanto os indicadores da dimensão negativa foram escalas que avaliaram afetos negativos, sintomas depressivos e traços de ansiedade e raiva. Os marcadores neuroendócrinos incluíram medidas de cortisol urinário e salivar, níveis de epinefrina e norepinefrina urinários, e concentração de Sulfato de Dehidroepiandrosterona (DHEA-S) sérico. O risco de doenças cardiovasculares foi avaliado a partir do peso, razão cintura/quadril, pressão sistólica e diastólica, colesterol HDL e total, e hemoglobinas glicosiladas. O principal achado deste estudo foi que os resultados de sete biomarcadores corroboram a hipótese de que as dimensões positivas e negativas da saúde mental possuem diferentes correlatos biológicos. Apenas os resultados de dois biomarcadores - peso e hemoglobina glicosilada - sustentam a hipótese do continuum bipolar.

\section{Considerações Finais}

O Bem-Estar Psicológico é um construto multidimensional que reflete características relativas ao funcionamento psicológico positivo ou ótimo. Ao basear-se na teoria psicológica, reúne avanços conceituais e achados empíricos de décadas de pesquisas nas áreas do desenvolvimento humano, psicologia humanista-existencial e saúde mental. Do ponto de vista metateórico, o BEP representa a tradição eudaimonica do estudo do bem-estar, resgatando o sentido original do bem-viver na doutrina aristotélica.

A operacionalização das dimensões do BEP permitiu o desenvolvimento de escalas psicométricas válidas e fidedignas para sua avaliação. Uma razoável quantidade de estudos demonstra evidências da validade dessas medidas, em suas diversas formas de 
apresentação. Entretanto, as possibilidades de avaliação do BEP não se limitam à abordagem quantitativa, mas ainda incluem métodos qualitativos, como a análise de narrativas.

Foram examinados achados que sugerem a associação do BEP a diversos processos positivos relacionados à saúde, não apenas no âmbito comportamental e cognitivo, com também no âmbito neuroendócrino. Esses achados encorajam pesquisadores da área a adotarem uma perspectiva biopsicossocial em suas investigações. O BEP é um construto importante para diferentes áreas da Psicologia (por exemplo: saúde, social, clínica, personalidade, desenvolvimento, avaliação psicológica), bem como para as ciências sociais e da saúde em geral. Foi observado que, no Brasil, durante o período de elaboração do presente manuscrito, apenas no estudo de Queroz e Neri (2005) o modelo de Bem-Estar Psicológico de Ryff foi citado. Dessa forma, constata-se a necessidade de outros estudos que incluam as dimensões do BEP na avaliação de indicadores de bem-estar, qualidade de vida e saúde.

\section{Referências}

An, J. S., \& Cooney, T. M. (2006). Psychological well-being in mid to late life: the role of generativity development and parent-child relationships across the lifespan. International Journal of Behavioral Development, 30 (5), 410-421.

Bauer, J. J., \& McAdams, D. P. (2004a). Growth goals, maturity and well-being. Developmental Psychology, 40 (1), 114-127.

Bauer, J. J., \& McAdams, D. P. (2004b). Personal growth in adults' stories of life transitions. Journal of Personality, 72 (3), 573-602.

Bauer, J. J., McAdams, C. P., \& Sakaedua, A. R. (2005). Interpreting the good life: growth memories in the lives of mature, happy people. Journal of Personality and Social Psychology, 88 (1), 203-217.

Bradburn, N. M. (1969). The structure of psychological wellbeing. Chicago: Aldine.

Burns, R. A., \& Machin, M. A. (2009). Investigating the structural validity of Ryff's psychological well-being scales across two samples. Social Indicators Research, 93 (2), 359-375.

Cheng, S. T., \& Chan, A. C. M. (2005). Measuring psychological well-being in the Chinese. Personality and Individual Differences, 38 (6), 1307-1316.

Clarke, P. J., Marshall, V. W., Ryff, C. D., \& Wheaton, B. (2001). Measuring psychological well-being in the Canadian Study of Health and Aging. International Psychogeriatrics,
Costa, P. T., \& McCrae, R. R. (1980). Influence of extraversion and neuroticism on subjective well-being: happy and unhappy people. Journal of Personality and Social Psychology, 38 (4), 668-678.

Diener, E. (1984). Subjective well-being. Psychological Bulletin, 95 (3), 542-575.

Diener, E., Suh, E. M., Lucas, R. E., \& Smith, H. L. (1999). Subjective well-being: three decades of progress. Psychological Bulletin, 125 (2), 276-302.

Diener, E., Suh, E. M., \& Oishi, S. (1997). Recent findings on subjective well-being. Indian Journal of Clinical Psychology, 24 (1), 25-41.

Fleeson, W., \& Baltes, P. B. (1996). The predictive power of perceived lifetime personality. Unpublished manuscript, Marx Plande Institure for Human Development and Education, Berlin.

Galinha, I., \& Ribeiro, J. L. P. (2005). História e evolução do conceito de bem-estar subjectivo. Psicologia, Saúde \& Doenças, 6 (2), 203-214.

Hayney, M. S., Love, G. D., Buck, J. M., Ryff, C. D., Singer, B., \& Muller, D. (2003). The association between psychosocial factors and vaccine-induced cytokine production. Vaccine, 21 (2), 2428-2432.

Holland, K. D., \& Holahan, C. K. (2003). The relation of social support and coping to positive adaptation to breast cancer. Psychology and Health, 18 (1), 15-29.

Jin, S., \& Moon, S. M. (2006). A study of well-being and school satisfaction among academically talented students attending a science high school in Korea. Gifted Child Quarterly, 50 (2), 169-184.

Kafka, G. J., \& Kozma, A. (2002). The construct validity of Ryff's scales of psychological well-being (SPWB) and their relationship to measures of subjective well-being. Social Indicators Research, 57 (2), 171-190.

Keyes, C. L. M. (2002). The mental health continuum: from languishing to flourishing in life. Journal of Health and Social Research, 43 (2), 207-222.

Keyes, C. L. M. (2006). Subjective well-being in mental health and human development research world wide: an introduction. Social Indicators Research, 77 (1), 1-10.

Keyes, C. L. M. (2007). Promoting and protecting mental health as flourishing: a complementary strategy for improving national mental health. American Psychologist, $62(2), 95-108$.

Keyes, C. L. M., \& Ryff, C. D. (2003). Somatization and mental health: a comparative study of the idiom of distress hypothesis. Social Science \& Medicine, 57 (10), 1833-1845.

Keyes, C. L. M., Shmotkin, D., \& Ryff, C. D. (2002). Optimizing well-being: the empirical encounter of two traditions. Journal of Personality and Social Psychology, 82 (6), 10071022.

Konow, J., \& Earley, J. (2008). The hedonistic paradox: is homo economicus happier? Journal of Public Economics, $92(2), 1-33$.

Larsen, R. J., Diener, E., \& Emmons, R. A. (1985). An evaluation of subjective well-being measures. Social Indicators Research, 17 (1), 1-18. 
Lindfors, P., \& Lundberg, U. (2002). Is low cortisol release an indicator of positive health? Stress and Health, 18 (4), 153-160.

Lindfors, P., Berntsson, L., \& Lundberg, U. (2006). Factor structure of Ryff's psychological well-being scales in Swedish female and male white-collar workers. Personality and Individual Differences, 40 (6), 1213-1222.

Marmot, M., Ryff, C. D., Bumpass, L. L., Shipley, M., \& Marks, N. F. (1997). Social inequalities in health: next questions and converging evidence. Social Science and Medicine, 44 (6), 901-910.

McCrae, R. R., \& Costa, P. T. (1989). More reasons to adopt the Five-Factor Model. American Psychologist, 44 (2), 451-453.

Neugarten, B. L., Havighurst, R., \& Tobin, S. (1961). The measurement of life satisfaction. Journal of Gerontology, $16,134-143$

Novo, R. F. (2003). Para além da eudaimonia: o bem-estar psicológico em mulheres na idade adulta avançada. Coimbra: Fundação Calouste Gulbenkian.

Queroz, N. C., \& Neri, A. L. (2005). Bem-estar psicológico e inteligência emocional entre homens e mulheres na meia-idade e na velhice. Psicologia: Reflexão e Crítica, 18 (2), 292-299.

Ruini, C., Ottolini, F., Rafanelli, C., Ryff, C. D., \& Fava, G. A. (2003). La validazione italiana delle Psychological Wellbeing Scales (PWB). Rivista di Psichiatria, 38 (3), 117-130.

Ryan, R. M., \& Deci, E. L. (2001). On happiness and human potentials: a review of research on hedonic and eudaimonic well-being. Annual Review of Psychology, 52, 141-166.

Ryff, C. D. (1989). Happiness is everything, or is it? Explorations on the meaning of psychological well-being. Journal of Personality and Social Psychology, 57 (6), 1069-1081.

Ryff, C. D., \& Essex, M. J. (1992). The interpretation of life experience and well-being: the sample case of relocation. Psychology and Aging, 7 (2), 507-517.

Ryff, C. D., \& Keyes, C. L. M. (1995). The structure of psychological well-being revisited. Journal of Personality and Social Psychology, 69 (4), 719-727.

Ryff, C. D., Keyes, C. L. M., \& Hughes, D. L. (2003). Status Inequalities, perceived discrimination, and eudaimonic well-being: do the challenges of minority life hone purpose and growth? Journal of Health and Social Behavior, 44 (3), 275-291.

Ryff, C. D., Love, G. D., Urry, H. L., Muller, D., Rosenkranz, M. A., Friedman, et al. (2006). Psychological well-being and ill-being: do they have distinct or mirrored biological correlates? Psychotherapy and Psychosomatics, 75 (2), 85-95.

Ryff, C. D., \& Singer B. H. (1998). The contours of positive human health. Psychological Inquiry, 9 (1), 1-28.

Ryff, C. D., \& Singer B. H. (2006). Best news yet on the six-factor model of well-being. Social Science Research, 35 (4), 1103-1119.

Ryff, C. D., \& Singer B. H. (2008). Know thyself and become what you are: a eudaimonic approach to psychological well-being. Journal of Happiness Studies, 9 (1), 13-39.

Ryff, C. D., Singer, B. H., \& Love, G. D. (2004). Positive health: connecting well-being with biology. Philosophical Transactions of the Royal Society B: Biological Sciences, 359 (29), 1383-1394.

Schmutte, P. S., \& Ryff, C. D. (1997). Personality and wellbeing: reexamining methods and meanings. Journal of Personality and Social Psychology, 73 (3), 549-559.

Sergrin, C., \& Taylor, M. (2007). Positive interpersonal relationships mediate the association between social skills and psychological well-being. Personality and Individual Differences, 43 (4), 637-646.

Sheldon, K. (2005). Positive value change during college: normative trends and individual differences. Journal of Research in Personality, 39 (2), 209-223.

Urry, H. L., Nitschke, J. B., Dolski, I., Jackson, D. C., Dalton, K. M., \& Mueller, C. J. (2004). Making a life worth living: neural correlates of wellbeing. Psychological Science, 15 (6), 367-372.

van Dierendonck, D. (2005). The construct validity of Ryff's scales of psychological well-being and its extension with spiritual well-being. Personality and Individual Differences, 36 (3), 629-643.

van Dierendonck, D., Díaz, D., Rodríguez-Carvajal, R., Blanco, A., \& Moreno-Jiménez, B. (2007). Ryff's six-factor model of psychological well-being, a Spanish exploration. Social Indicators Research, 87 (3), 473-479.

Vleioras, G., \& Bosma, H. (2005). Are identity styles important for psychological well-being? Journal of Adolescence, 28 (3), 397-409.

Waterman, A. S. (1993). Two conceptions of happiness: contrasts of personal expressiveness (eudaimonia) and hedonic enjoyment. Journal of Personality and Social Psychology, 64 (4), 678-691.

Recebido em: 16/7/2010

Versão final em: 3/2/2012

Aprovado em: 13/2/2012 